\title{
Helicitogenesis: WIMPy baryogenesis with sterile neutrinos and other realizations
}

\section{J. Racker and N. Rius}

Departamento de Física Teórica,

Instituto de Física corpuscular (IFIC), Universidad de Valencia-CSIC,

Edificio de Institutos de Paterna, Apt. 22085, 46071 Valencia, Spain

E-mail: racker@ific.uv.es, nuria@ific.uv.es

ABSTRACT: We propose a mechanism for baryogenesis from particle decays or annihilations that can work at the TeV scale. Some heavy particles annihilate or decay into a heavy sterile neutrino $N$ (with $M \gtrsim 0.5 \mathrm{TeV}$ ) and a "light" one $\nu$ (with $m \ll 100 \mathrm{GeV}$ ), generating an asymmetry among the two helicity degrees of freedom of $\nu$. This asymmetry is partially transferred to Standard Model leptons via fast Yukawa interactions and reprocessed into a baryon asymmetry by the electroweak sphalerons. We illustrate this mechanism in a WIMPy baryogenesis model where the helicity asymmetry is generated in the annihilation of dark matter. This model connects the baryon asymmetry, dark matter, and neutrino masses. Moreover it also complements previous studies on general requirements for baryogenesis from dark matter annihilation. Finally we discuss other possible realizations of this helicitogenesis mechanism.

KeYwords: Cosmology of Theories beyond the SM, Beyond Standard Model

ARXIV EPRINT: 1406.6105 


\section{Contents}

1 Introduction 1

2 The mechanism and a WIMPy leptogenesis model 3

3 Dynamics and evolution equations for the helicity asymmetry $\quad 7$

4 Other realizations of helicitogenesis $\quad \mathbf{1 0}$

4.1 WIMPy leptogenesis with spontaneous $\mathrm{U}(1)_{L}$ symmetry breaking $\quad 10$

$\begin{array}{lll}4.2 & \text { Helicitogenesis in WIMP decay } & 13\end{array}$

5 Conclusions and outlook $\quad 14$

\section{Introduction}

The nature of the Dark Matter (DM) and the origin of the Baryon Asymmetry of the Universe (BAU) are unknown and both require physics beyond the Standard Model (SM) to be explained. Also puzzling -or maybe a hint?- is the fact that the energy densities are comparable, $\Omega_{D M} \sim 5 \Omega_{B}$ [1]. The conventional explanations are unrelated, and often involve very different scales of new physics. The most popular candidates for DM are Weakly Interacting Massive Particles (WIMPs), which provide the so-called "WIMP miracle": the thermal relic abundance of a stable WIMP is naturally of the order of the observed $\Omega_{D M}$. Regarding the baryon abundance, the Sakharov conditions to generate dynamically the BAU can be fulfilled in a variety of extensions of the SM at very different energy scales, ranging from below the electroweak to the Planck scale. However the similarity of the DM and baryonic energy densities suggests a common origin, and such possibility has been extensively studied in recent years.

Most models relating the dark and baryonic matter abundances involve Asymmetric Dark Matter (ADM), i.e., the DM we observe today is due to a particle-antiparticle asymmetry in the dark sector which is somehow tied to the one in baryons (for extensive reviews see $[2,3])$. However in the ADM scenario the "WIMP miracle" is lost. This has motivated several attempts to find a mechanism that preserves the natural DM relic density of a WIMP and at the same time relates the dark and baryonic matter abundances $[4,5]$. One possibility is the existence of several WIMPs, at least one of them stable which will make up the DM of the Universe and other(s) long-lived, which will generate the BAU in their out of equilibrium decay $[6,7]$. In a more minimalistic mechanism, dubbed WIMPy Baryogenesis (WB), the BAU is generated directly in the annihilation of a stable WIMP [8]. The phenomenology of several WB models has been studied in [9] and conditions for generating the observed $\Omega_{B}$ and $\Omega_{D M}$ via this mechanism analyzed in detail in [10]. 
One of the challenges of $\mathrm{WB}$ is that the BAU must be produced at temperatures $T \sim m_{\chi}$-with $m_{\chi}$ the DM mass- which are very low for thermal baryogenesis mechanisms (usually $m_{\chi} \lesssim$ few $\mathrm{TeV}$ and in any case $m_{\chi}<340 \mathrm{TeV}$ [11]). At such temperatures the processes responsible for the $\mathrm{CP}$ even phase in the reaction generating the BAU are typically very fast compared to the Hubble rate, and therefore they erase most of the cosmic asymmetry (for recent detailed discussions on this subject see [10, 12]). One way out of this problem is to include massive particles so that the washout processes decouple exponentially. To the best of our knowledge this idea was first fully explored in [8] for baryogenesis from DM annihilations, and studied in [12] for baryogenesis from particle decays. In these works the annihilations -or decays- directly produced a baryon or lepton asymmetry in SM fields, and given that the mediators of the annihilations -or the decaying particles- were singlets, the massive fields had some SM charges and could also store an asymmetry. In turn this asymmetry had to vanish exponentially without canceling the SM baryon -or lepton- asymmetry, which lead to some complications in the implementation of the mechanism (as the need for a light sterile dark sector or a very fast interaction violating some of the charges of the massive field).

In this work we propose a variation of the above scenario: the massive particles responsible for the exponential suppression of the washout are Majorana fermions and therefore they do not have any conserved charge, avoiding the complications just mentioned. It can be realized in both, baryogenesis from DM freeze out and from heavy particle out-ofequilibrium decay. We illustrate this mechanism in a WB model where the DM annihilates into sterile neutrinos, which in turn are responsible for neutrino masses via the type I seesaw. In this way we address the possibility of relating the DM and BAU problems with yet another puzzle that requires physics beyond the SM: neutrino masses. Furthermore our work complements previous studies [10] on general conditions for having baryogenesis from DM annihilation.

The basic requirement for generating the BAU is the existence of heavy sterile neutrinos $N(M \gtrsim 0.5 \mathrm{TeV})$ and also lighter ones $\nu$ (with $m \ll 100 \mathrm{GeV}$ ), both of them interacting with the DM via SM singlet scalars. WIMP annihilations to the sterile neutrinos violate CP, generating an asymmetry among the two helicity degrees of freedom of $\nu$. This asymmetry is transferred to SM leptons via fast Yukawa interactions, which should be in equilibrium prior to the electroweak phase transition to ensure that a baryon asymmetry is also induced by the sphaleron $(B+L)$-violating interactions. Moreover, since no asymmetry accumulates in the heavy sector $N$, some of the requisites of the original WB models -a $Z_{4}$ symmetry and a light sterile dark sector- are automatically avoided. Note that in the limit $m \rightarrow 0$ there is a global U(1) symmetry. This implies the existence of a -perturbatively- conserved lepton number which coincides with the helicity for the $\nu$ 's, i.e. a $\nu$ with positive (negative) helicity has lepton number $L_{\nu}=1(-1)$.

Given that the mass scale of the sterile neutrinos is unconstrained and its origin unknown, it is worthwhile to explore the consequences of having several mass scales in the sterile sector without any theoretical prejudice. Therefore we first adopt a purely phenomenological perspective, illustrating the proposed mechanism by means of a minimal model.

It is however tempting to justify the hierarchical sterile neutrino mass spectrum by some broken, global or local, symmetry. Moreover, there are neutrino mass models in 
which this is actually the case, as in the double seesaw scenario [13]. We then discuss a realization of the "helicitogenesis" mechanism within the double seesaw framework, in a model with spontaneously broken $\mathrm{U}(1)_{L}$ symmetry $\left(\mathrm{U}(1)_{B-L}\right.$ in the gauged case), in which the DM is charged under lepton number.

Notice that a similar helicity asymmetry in the SM-singlet Majorana neutrinos, also transferred to the SM lepton sector by fast Yukawa interactions, is the basis of baryogenesis via neutrino oscillations originally proposed in $[14,15]$. In such case the helicity asymmetry is due to the CP-violating coherent neutrino oscillations, while in our scenario it is generated in the CP-violating DM annihilation or CP-violating decay of a heavy particle. Therefore the requirements $m \ll 100 \mathrm{GeV}$ and some sterile-active neutrino Yukawa interactions in equilibrium before the electroweak phase transition are common to all mechanisms, while we have extra sources of $\mathrm{CP}$ violation.

The paper is organized as follows. In section 2 we describe the basics of the helicitogenesis mechanism and implement it in a minimal WB model. In section 3 we write the set of Boltzmann Equations (BEs) relevant for the generation and evolution of the helicity asymmetry in the sterile neutrinos. Section 4 is devoted to other realizations of helicitogenesis in the context of a neutrino mass model with spontaneous $\mathrm{U}(1)_{L}$ symmetry breaking, and we conclude in section 5 .

\section{The mechanism and a WIMPy leptogenesis model}

In WB [8] the DM, $\chi$, is a weakly interacting massive particle whose relic density is determined by the freeze out of some annihilation process $\chi \chi \rightarrow \bar{\Psi} f$, with $f$ a SM fermion and $\Psi$ a heavy exotic particle. The amplitude for the process $\chi \chi \rightarrow \bar{\Psi} f$ contains a CP odd phase coming from complex couplings and a $\mathrm{CP}$ even phase from the absorptive part of one loop contributions, therefore it violates CP. Moreover, depending on whether $f$ is a SM quark or a lepton, the interaction $\chi \chi \rightarrow \bar{\Psi} f$ violates SM B or L, respectively. In this way all Sakharov conditions are satisfied and some baryon or lepton asymmetry is produced in the annihilation of DM. If $\Psi$ is heavy enough, $m_{\Psi} \gtrsim m_{\chi}$ [8], the processes that can potentially washout the asymmetry -most notably $\bar{\Psi} f \leftrightarrow \Psi \bar{f}$ - are Boltzmann suppressed, hence a significant amount of matter asymmetry may survive.

However, the annihilation $\chi \chi \rightarrow \bar{\Psi} f$ also generates an asymmetry in the $\Psi$ sector and it is not trivial to avoid a cancellation of the total matter asymmetry after $\Psi$ disappears from the thermal bath. This seems to force into building more complicated models. E.g. in the original work [8] the $\Psi$ decay into a light hidden sector, while decays into SM particles are forbidden by a $Z_{4}$ symmetry. Then in [10] it was shown that WB could work without a light hidden sector or a discrete $Z_{4}$ symmetry, but still the problem associated to the asymmetry in $\Psi$ had to be solved complicating the models in some other ways. Here we present a WIMPy model where the role of $\Psi$ is played by heavy Majorana fermions (subsequently called $N_{i}$ ) which lack a conserved charge to store asymmetry. ${ }^{1}$ Therefore no

\footnotetext{
${ }^{1} \mathrm{~A}$ massive real scalar could also be used to suppress the dangerous washouts in a similar way, but a different baryogenesis model would be necessary to implement this option.
} 
additional fields beyond those participating in the annihilation of DM must be added. In addition, this model yields a close connection to light neutrino masses.

The SM is extended with some singlet real scalars, $S_{a}$, and Majorana fermions, $\chi, N_{i}, \nu_{j}$, with $\{a, i, j=1, \ldots\}$, together with a discrete $Z_{2}$ symmetry to ensure the stability of the DM. The DM candidate $\chi$ is the only odd particle under $Z_{2}$. It could also be a Dirac singlet, but we choose it Majorana to minimize the number of new degrees of freedom. The $N_{i}$ and $\nu_{j}$ are sterile neutrinos with "high" $\left(M_{i} \sim \mathcal{O}(\mathrm{TeV})\right)$ and "low" $\left(m_{j} \ll 100 \mathrm{GeV}\right)$ masses, respectively. ${ }^{2}$ In the basis which yields a diagonal Majorana mass matrix with real and positive entries, the most general renormalizable Lagrangian with the given fields and symmetries reads

$$
\begin{aligned}
-L= & -L_{\mathrm{SM}}-L_{\mathrm{kin}}+V\left(S_{a}, H\right) \\
& +\frac{1}{2}\left\{m_{\chi} \bar{\chi} \chi+M_{i} \bar{N}_{i} N_{i}+m_{j} \bar{\nu}_{j} \nu_{j}\right\} \\
& +\frac{1}{2}\left\{\lambda_{\chi a} S_{a} \bar{\chi} P_{R} \chi+\lambda_{N a i j} S_{a} \bar{N}_{i} P_{R} N_{j}+\lambda_{\nu a i j} S_{a} \bar{\nu}_{i} P_{R} \nu_{j}+\text { h.c. }\right\} \\
& +\left\{\lambda_{a i j} S_{a} \bar{N}_{i} P_{R} \nu_{j}+h_{N \alpha i} \tilde{H} \bar{\ell}_{\alpha} P_{R} N_{i}+h_{\nu \alpha j} \tilde{H} \bar{\ell}_{\alpha} P_{R} \nu_{j}+\text { h.c. }\right\},
\end{aligned}
$$

where there is an implicit sum over repeated family indices, $\ell_{\alpha}$ are the leptonic $\mathrm{SU}(2)$ doublets, $H$ is the Higgs field ( $\widetilde{H}_{2}=i \tau_{2} H_{2}^{*}$, with $\tau_{2}$ Pauli's second matrix), $V\left(S_{a}, H\right)$ is the scalar potential and $P_{R, L}=\left(1 \pm \gamma_{5}\right) / 2$ are the chirality projectors. Latin indices denote sterile neutrinos while Greek indices refer to the SM lepton doublets. All Majorana fields $\xi\left(\xi=\chi, N_{i}, \nu_{j}\right)$ satisfy $\xi^{c}=\lambda_{\xi} \xi$, with $\lambda_{\xi}$ a phase factor. Notice that the Yukawa matrices $\lambda_{N a}, \lambda_{\nu a}$ are symmetric.

The key for having baryogenesis in this model is that $m_{j} \ll T_{\text {sfo }}$ at least for one species $\nu_{j}$, where $T_{\text {sfo }}=\mathcal{O}(100 \mathrm{GeV})$ is the temperature at which the electroweak sphalerons freeze out. Then an asymmetry among the two helicity degrees of freedom of $\nu_{j}, \nu_{j}^{+}$and $\nu_{j}^{-}$, can be generated from the annihilation of DM $\chi \chi \rightarrow N_{i} \nu_{j}$, in the same way as the lepton or baryon asymmetry is created from $\chi \chi \rightarrow \bar{\Psi} f$ in previous WB models [8-10]. As long as some of the Yukawa couplings of $\nu_{j}, h_{\nu \alpha j}$, are large enough, the helicity asymmetry in the $\nu_{j}$ is efficiently transferred to the SM lepton sector. In turn, this is partially transformed into a baryon asymmetry by the sphaleron processes. Once these decouple at $T_{\text {sfo }}$, the $\mathrm{BAU}$ is frozen. Notice that if $m_{j} \neq 0$, the helicity depends on the reference frame. We will be always working in the thermal bath rest frame.

This baryogenesis scenario requires at least one species of $N^{\prime} s$ and $\nu^{\prime} s$, and two real scalars to have CP violation, $S_{1}$ and $S_{2}$ (actually we will explain later that there can be a CP odd phase with just one scalar, contrary to previous WB models, but the amount of $\mathrm{CP}$ violation is most likely too small to have successful baryogenesis). Next we indicate the approximate range of values that the parameters in the Lagrangian (2.1) can take for this baryogenesis mechanism to be successful.

\footnotetext{
${ }^{2}$ Although in this model the species $\nu_{j}$ and $N_{i}$ differ only in their masses, we denote them by different symbols to emphasize their distinct roles for leptogenesis.
} 
- $m_{\chi}$ : to generate the asymmetry before sphalerons freeze out, the DM has to start annihilating well above $T_{\text {sfo }}$, hence $m_{\chi} \gtrsim 1 \mathrm{TeV}$.

- $m_{S a}$ : although the asymmetry could also be produced in the decays of $S_{a}$ (see [8]), we are interested in the case that the asymmetry is mainly produced in the annihilation of DM, hence we impose that the masses of the singlet scalars, $m_{S a}$, are $m_{S a} \gtrsim m_{\chi}$ (in this way the CP conserving annihilation channel $\chi \chi \rightarrow S_{a} S_{a}$ is negligible).

- $M_{i}$ : the heavy $N_{i}$ are introduced to have a Boltzmann suppression $e^{-M_{i} / T}$ of washouts that can be very fast when baryogenesis occurs at low temperatures (see [8] and [10, 12] for detailed discussions on this point). For this Boltzmann suppression to be efficient $M_{i} \gtrsim(0.5-1) m_{\chi}$. In addition, $M_{i}<2 m_{\chi}$ to allow for DM annihilations when $\chi$ becomes non-relativistic.

- $m_{j}$ : to create an helicity asymmetry in the $\nu$-sector it is necessary that $m_{j} \ll T_{\text {sfo }}$. In section 3 this issue will be studied in more detail.

- $\lambda_{\chi a}, \lambda_{a i j}$ : these couplings must be $\mathcal{O}(1)$ for having enough $\mathrm{CP}$ violation and a correct DM relic abundance. More precisely, it is the imaginary part of $\lambda_{\chi a}$ that has to be large, so that there is a sufficiently fast, not velocity-suppressed annihilation rate.

- $\lambda_{\nu a i j}$ : they induce washouts of the helicity asymmetry that are not Boltzmannsuppressed, e.g. via the reaction $\nu_{j}^{+} \nu_{j}^{+} \leftrightarrow \nu_{j}^{-} \nu_{j}^{-}$. For these processes to be slow enough $\left|\lambda_{\nu a i j}\right| m_{\chi} / m_{S a} \ll 10^{-3}$.

- $\lambda_{N a i j}$ : they do not play an important role because the corresponding processes are Boltzmann suppressed, hence they are unconstrained.

- $h_{\nu \alpha j}$ : it is crucial that there be at least one fast Yukawa interaction between the $\nu_{j}$ and $\ell_{\alpha}$, and therefore at least one coupling $h_{\nu \alpha j} \gtrsim 2 \times 10^{-7}[16,17]$.

- $h_{N \alpha i}$ : they mediate washout processes like $\ell_{\alpha} H \leftrightarrow \bar{\ell}_{\alpha} \bar{H}$, which should be slow at $T \gtrsim$ $T_{\text {sfo. }}$ As for the $\lambda_{\nu a i j}$ couplings, this requirement is satisfied for $\left|h_{N \alpha i}\right| m_{\chi} / M_{i} \ll 10^{-3}$.

In addition the heavy singlet sector must be populated at $T \gtrsim m_{\chi}$. This can be achieved by some fast interaction connecting the sterile and SM sectors, like one among the $S_{a}$ and the Higgs or the Yukawa interactions between the $N_{i}$ and $\ell_{\alpha}$.

The allowed region in the parameter space of $m_{\chi}, m_{S a}, M_{i}, \lambda_{\chi a}$ and $\lambda_{a i j}$ is very similar to previous models of WB [8-10] (with $N_{i}$ playing the role of the heavy exotic annihilation product); very roughly it consists of masses above $\sim 1 \mathrm{TeV}$ and $\mathcal{O}(1)$ couplings. On the other hand, if helicitogenesis occurs in the decay of the lightest scalar, $S_{1}$, the conditions for generating the $\nu$ helicity asymmetry are analogous to those for standard leptogenesis at the TeV scale, basically $\lambda_{1 i j} \lesssim 10^{-7}, m_{S a} \gtrsim 1 \mathrm{TeV}$, and $M_{i} \gtrsim 0.5 \mathrm{TeV}$ [12]. Hence we are not going to develop these issues further. Instead we will concentrate on the constraints imposed by leptogenesis via helicitogenesis and the connection with light neutrino masses: 
(i) The N's and $\nu$ 's must decay before Big Bang Nucleosynthesis (BBN) to avoid observational constraints.

This requirement is not difficult to accomplish for the heavy neutrinos $N_{i}$. One possibility is that $h_{N \alpha i}$ be non-negligible to allow for the decay $N_{i} \rightarrow \ell_{\alpha} H$, but at the same time small enough for the washout processes like $\ell_{\alpha} H \leftrightarrow \bar{\ell}_{\alpha} \bar{H}$ to be slow at $T \gtrsim T_{\text {sfo }}$. This condition is easy to satisfy given that the rate of this last process is $\propto h_{N \alpha i}^{4}$, while the rate of the former is $\propto h_{N \alpha i}^{2}$. Another possibility could be to choose $\lambda_{\nu a i j}$ large enough to induce three body decays like $N_{i} \rightarrow \nu_{j} \nu_{j} \nu_{j}$, but not as large as to have fast washouts $\nu_{j}^{+} \nu_{j}^{+} \leftrightarrow \nu_{j}^{-} \nu_{j}^{-}$(again note that the rate of $\nu_{j}^{+} \nu_{j}^{+} \rightarrow \nu_{j}^{-} \nu_{j}^{-}$is $\propto \lambda_{\nu a j j}^{4}$ while the rate of $N_{i} \rightarrow \nu_{j} \nu_{j} \nu_{j}$ is $\left.\propto \lambda_{\nu a j j}^{2}\right)$.

The main decay modes of the (lightest) $\nu_{j}$ are $\nu_{j} \rightarrow \nu_{\alpha} \nu_{\beta} \bar{\nu}_{\beta}, \nu_{j} \rightarrow \nu_{\alpha} e_{\beta}^{-} e_{\beta}^{+}, \nu_{j} \rightarrow$ $\nu_{\alpha} q_{\beta} \bar{q}_{\beta}$, via $Z$ exchange, $\nu_{j} \rightarrow e_{\alpha}^{-} e_{\beta}^{+} \nu_{\beta}, \nu_{j} \rightarrow e_{\alpha}^{-} q_{\beta} \bar{q}_{\beta}^{\prime}$ via $W$ exchange, and the corresponding $\mathrm{CP}$-conjugate processes, where $e_{\alpha}$ denotes the charged leptons $e, \mu, \tau$ and $q_{\beta}$ stands for the SM quarks, except the top. The $\nu_{j}$ decay width is given by

$$
\Gamma_{j}=\frac{G_{F}^{2} m_{j}^{3}}{192 \pi^{3}} \sum_{\alpha, \beta} A_{\alpha \beta}\left|h_{\nu \alpha j} v\right|^{2},
$$

where the sum extends over the kinematically allowed decay channels, $v=\langle H\rangle=$ $174 \mathrm{GeV}$ is the Higgs vev, $G_{F}$ is the Fermi constant, and $A_{\alpha \beta}$ are $\mathcal{O}(1)$ coefficients that depend on the number of degrees of freedom associated to each mode. Using the above equation, with at least one $h_{\nu \alpha j} \sim 10^{-7}$ we find that $m_{j} \gtrsim 1 \mathrm{GeV}$, in order for $\nu_{j}$ to decay before BBN.

Moreover, even if the decay of the $\nu_{j}$ occurs safely before BBN, it may lead to an increase of entropy density after the electroweak phase transition, which would dilute the baryon asymmetry. We have checked that this entropy increase is negligible for $m_{j} \gtrsim 10 \mathrm{GeV}$, when the $\nu_{j}$ decays at $T \sim 500 \mathrm{MeV}$, before the QCD phase transition. However it can be a concern for lower masses, $m_{j} \sim 1 \mathrm{GeV}$. In this case, the decay occurs after the QCD phase transition and the increase in entropy density can be up to order 10. This implies that the baryon asymmetry originally produced should be an order of magnitude larger, which could require Yukawa couplings close to the perturbative limit.

(ii) Constraints from neutrino masses.

The seesaw contribution of $\nu_{j}$ to the light neutrino masses is given by $\left(m_{L}\right)_{\alpha \beta} \sim$ $h_{\nu \alpha j} h_{\nu \beta j} \frac{v^{2}}{m_{j}}$. Taking into account that $h_{\nu \alpha j} \gtrsim 2 \times 10^{-7}$, one has that $\left(m_{L}\right)_{\alpha \beta}[\mathrm{eV}] \gtrsim$ $1 / m_{j}[\mathrm{GeV}]$. The strongest constraints on the absolute scale of neutrino masses are derived from cosmological observations, via their contribution to the energy density of the Universe and the growth of structure [1]. Since these bounds are very sensitive to the assumptions about the expansion history of the Universe and to the data included in the analysis, we choose the conservative upper bound on the sum of light neutrinos masses of roughly $1 \mathrm{eV}$, obtained by combining $\mathrm{CMB}$ and large scale structure data when including several departures from the $\Lambda$ CDM model [18]. This bound implies 
that $m_{j} \gtrsim 3 \mathrm{GeV}$, unless there is a fine tuning among the phases of the Yukawa couplings of different species of $\nu^{\prime} s$, so that they give big contributions to $\left(m_{L}\right)_{\alpha \beta}$ with opposite signs that cancel each other. The atmospheric mass scale, $0.05 \mathrm{eV}$, can be naturally obtained with $m_{j} \sim 20 \mathrm{GeV}$, thus the two conditions for our mechanism to work, $m_{j} \ll T_{\text {sfo }} \sim 100 \mathrm{GeV}$ and $h_{\nu \alpha j} \gtrsim 2 \times 10^{-7}$ are compatible with the observed light neutrino masses. Notice that these two requirements are also needed when the helicity asymmetry in the singlet neutrinos is generated via neutrino oscillations [14].

Analogously, the heavy singlets $N_{i}$ also contribute to light neutrino masses an amount $h_{N \alpha i} h_{N \beta i} \frac{v^{2}}{M_{i}}$. Barring accidental cancellations, $h_{N} \lesssim\left(10^{-5}-10^{-6}\right) \sqrt{M_{i} / 1 \mathrm{TeV}}$ is consistent with present data, and also allows for the $N_{i}$ decay before BBN.

One may worry that the separation of the mass scales $m_{j} \ll M_{i}$ is not stable under radiative corrections, since there is not any symmetry protecting the small masses. In fact, $\nu_{j}$ self-energy diagrams with virtual $S_{a}$ and $N_{i}$ will induce Majorana masses for the $\nu_{j}$ at one loop of order

$$
m^{1-\text { loop }} \sim \frac{\left(\lambda_{a i j}\right)^{2}}{16 \pi^{2}} M_{i} \log \left(\frac{M_{i}^{2}}{m_{S a}^{2}}\right) .
$$

Thus for $\lambda_{a i j}$ of $\mathcal{O}(1)$, generically required for WB, we expect $m^{1-\text { loop }} \sim 10^{-2} M_{i}$, which does not upset the condition $m \ll 100 \mathrm{GeV}$ for $M_{i}$ of order few TeV. Indeed, this loop contribution is naturally of the correct size for helicitogenesis to work.

Notice that since $S_{a}$ are real scalar singlets, the potential $V\left(S_{a}, H\right)$ in eq. (2.1) will contain trilinear terms of the form $\mu_{a} S_{a} H^{\dagger} H$. After electroweak symmetry breaking such terms induce vevs $\left\langle S_{a}\right\rangle \neq 0$, which in turn generate a mixing among the light, $\nu$, and heavy, $N$, neutrino species. We assume that the $\mu_{a}$ parameters are not too large, therefore the corrections to the $\nu$ masses still satisfy the requirement $\Delta m \sim\left(\lambda_{a i j}\left\langle S_{a}\right\rangle\right)^{2} / M_{i} \ll T_{\text {sfo }}$ and the mixing does not affect the proposed baryogenesis mechanism.

\section{Dynamics and evolution equations for the helicity asymmetry}

In this section we will show how to calculate the helicity asymmetry in the $\nu_{j}$ sector and its partial transformation into a baryon asymmetry. For simplicity we will consider only one species of $N^{\prime} s$ and $\nu^{\prime} s, N \equiv N_{1}$ and $\nu \equiv \nu_{1}$, and hence we will omit the indices associated with the $\nu$ and $N$ sectors. As noted in [19], in the thermal bath rest frame isotropy implies that the spin density matrix is diagonal in the helicity basis. This allows to write a set of BEs for the populations of $\nu^{+}$and $\nu^{-}$involving no coherences. Actually, the quantity of interest is the helicity asymmetry $Y_{\Delta \nu} \equiv Y_{\nu^{+}}-Y_{\nu^{-}}$, where for any particle $X$ we define $Y_{X} \equiv n_{X} / s$ as the number density of $X$ normalized to the entropy density. ${ }^{3}$

The asymmetry $Y_{\Delta \nu}$ originates from interactions in the singlet sector of the model. In turn, this asymmetry is partially transferred to the lepton sector via the Yukawa interactions among $\nu$ and the SM lepton doublets. Finally the electroweak sphalerons transform

\footnotetext{
${ }^{3}$ The population of $\nu$ 's is in kinetic equilibrium due to different fast processes like scatterings with the $N$ 's and Yukawa interactions with SM leptons. This allows for an easy integration of the momentum degrees of freedom, leading to simple Boltzmann equations for the number densities.
} 
part of the lepton asymmetry into a baryon one. A fairly good approximation is to consider that these different stages do not happen simultaneously: first $Y_{\Delta \nu}$ is generated while the DM annihilations freeze out and only then the Yukawa interactions and sphalerons act to get the final BAU. In other words, we neglect spectator processes during the generation of the helicity asymmetry. From the results of [20] we expect that this type of approximation is accurate within factors not larger than $\sim 2$. We will also assume that thanks to the $N_{i}$-interactions described in the previous section, $Y_{N_{i}}$ follows an equilibrium distribution while the DM is annihilating. Then $Y_{\Delta \nu}$ can be obtained from the following set of BEs (the details of the derivation of these BEs are very similar to those described in, e.g., the appendix B of [10]):

$$
\begin{aligned}
s z H(z) \frac{\mathrm{d} Y_{\Delta \nu}}{\mathrm{d} z}= & \left(\frac{Y_{\chi}^{2}}{Y_{\chi}^{e q 2}}-1\right) \epsilon \gamma(\chi \chi \rightarrow \nu N)-\frac{Y_{\Delta \nu}}{Y_{\nu}^{e q}}\left[2 \gamma\left(\nu^{+} N \rightarrow \nu^{-} N\right)\right. \\
& \left.+\gamma\left(\chi \chi \rightarrow \nu^{+} N\right)-4 \gamma\left(\nu^{+} \nu^{+} \rightarrow N N\right)-\frac{Y_{\chi}}{Y_{\chi}^{e q}} \gamma\left(\chi \nu^{+} \rightarrow \chi N\right)\right], \\
s z H(z) \frac{\mathrm{d} Y_{\chi}}{\mathrm{d} z}= & -2\left[\frac{Y_{\chi}^{2}}{Y_{\chi}^{e q 2}}-1\right] \gamma(\chi \chi \rightarrow \nu N) .
\end{aligned}
$$

Here $z \equiv m_{\chi} / T$ and $H(z)$ is the Hubble rate. The reaction density $\gamma(a, b \rightarrow c, d)$ is the number of $a, b \rightarrow c, d$ processes per unit time and volume, summing over all the degrees of freedom of the particles involved, including the helicity, unless this one is explicitly specified, as e.g. in $\gamma\left(\chi \chi \rightarrow \nu^{+} N\right)$ which only involves $\nu$ 's with positive helicity. ${ }^{4}$ It is given by

$$
\gamma(a, b \rightarrow c, d)(z)=\frac{m_{\chi}^{4}}{64 \pi^{4} z} \int_{x_{\min }}^{\infty} d x \sqrt{x} \sigma_{R}\left(x m_{\chi}^{2}\right) K_{1}(z \sqrt{x}),
$$

where $x \equiv s / m_{\chi}^{2}, x_{\min }=\operatorname{Max}\left\{\left(\frac{m_{a}+m_{b}}{m_{\chi}}\right)^{2},\left(\frac{m_{c}+m_{d}}{m_{\chi}}\right)^{2}\right\}$, and here $s$ is the center of mass energy squared. ${ }^{5}$ The reduced cross section $\sigma_{R}$ is related to the total cross section $\sigma$ via

$$
\sigma_{R}(s)=\frac{2 \lambda\left(s, m_{a}^{2}, m_{b}^{2}\right)}{s} \sigma(s) \quad \text { and } \quad \lambda\left(s, m_{a}^{2}, m_{b}^{2}\right) \equiv\left(s-\left(m_{a}+m_{b}\right)^{2}\right)\left(s-\left(m_{a}-m_{b}\right)^{2}\right) .
$$

In the eq. (3.1) we have neglected the CP asymmetry in the decay of $S_{a}$, which is a good approximation if $m_{S_{a}} \gtrsim 2 m_{\chi}$ because that asymmetry would be washed out very efficiently. Hence the helicity asymmetry is generated mainly in the annihilation of DM and the CP asymmetry per annihilation appearing in eq. (3.1), $\epsilon$, is defined as

$$
\epsilon \equiv \frac{\Delta \gamma(\chi \chi \rightarrow \nu N)}{\gamma(\chi \chi \rightarrow \nu N)}
$$

\footnotetext{
${ }^{4}$ Note that the $N$ 's are non-relativistic in the relevant epoch for baryogenesis, hence the chiral operators $P_{R} N$ and $P_{L} N$ in eq. (2.1) can create and destroy any helicity state of $N$. Therefore the helicity of the heavy sterile neutrinos does not play a major role and the rates are defined summing over the spin degree of freedom of $N$.

${ }^{5}$ We use the symbol $s$ both for the entropy density and for the center of mass energy squared. However it is always clear from the context which quantity we are referring to.
} 
where $\Delta \gamma(\chi \chi \rightarrow \nu N)=\gamma\left(\chi \chi \rightarrow \nu^{+} N\right)-\gamma\left(\chi \chi \rightarrow \nu^{-} N\right)$ and $\gamma(\chi \chi \rightarrow \nu N)$ is the total annihilation rate, $\gamma(\chi \chi \rightarrow \nu N)=\gamma\left(\chi \chi \rightarrow \nu^{+} N\right)+\gamma\left(\chi \chi \rightarrow \nu^{-} N\right)$. As long as there are at least two species of scalars, $S_{1}$ and $S_{2}$, there is a contribution to the CP asymmetry at zeroth order in $m / m_{S_{a}}$ (where $m \equiv m_{1}$ ). Up to $\mathcal{O}(1)$ numerical factors, the reduced cross sections relevant for the calculation of the $\mathrm{CP}$ asymmetry, in the limit $m \rightarrow 0$, are given by [10]:

$$
\begin{aligned}
& \Delta \sigma_{R}(\chi \chi\rightarrow \nu N) \\
&=\frac{1}{8 \pi^{2}} \frac{\sqrt{s-4 m_{\chi}^{2}}}{s^{3 / 2}\left(s-m_{S_{1}}^{2}\right)\left(s-m_{S_{2}}^{2}\right)} \\
& \quad \times\left\{2 \lambda_{\chi 1} \lambda_{\chi 2} \operatorname{Im}\left(\lambda_{1} \lambda_{2}^{*}\right)\left(\left|\lambda_{1}\right|^{2} \frac{f_{S}\left(m_{S_{1}}\right)+f_{V}\left(m_{S_{1}}\right)}{s-m_{S_{1}}^{2}}-\left|\lambda_{2}\right|^{2} \frac{f_{S}\left(m_{S_{2}}\right)+f_{V}\left(m_{S_{2}}\right)}{s-m_{S_{2}}^{2}}\right)\right. \\
&\left.\quad-\operatorname{Im}\left(\lambda_{1}^{2} \lambda_{2}^{* 2}\right)\left(\lambda_{\chi 1}^{2} \frac{f_{S}\left(m_{S_{2}}\right)+f_{V}\left(m_{S_{2}}\right)}{s-m_{S_{1}}^{2}}-\lambda_{\chi 2}^{2} \frac{f_{S}\left(m_{S_{1}}\right)+f_{V}\left(m_{S_{1}}\right)}{s-m_{S_{2}}^{2}}\right)\right\},
\end{aligned}
$$

and

$$
\sigma_{R}(\chi \chi \rightarrow \nu N)=\frac{1}{8 \pi} s^{1 / 2} \sqrt{s-4 m_{\chi}^{2}}\left(s-M^{2}\right)\left|\frac{\lambda_{\chi 1} \lambda_{1}}{\left(s-m_{S_{1}}^{2}\right)}+\frac{\lambda_{\chi 2} \lambda_{2}}{\left(s-m_{S_{2}}^{2}\right)}\right|^{2},
$$

where $\lambda_{a} \equiv \lambda_{a 11}$. The loop functions, $f_{S}\left(m_{S_{a}}\right)$ and $f_{V}\left(m_{S_{a}}\right)$ (that also depend on $M \equiv$ $M_{1}$ ), as well as the remaining density rates in eq. (3.1), can be found in appendix A of [10].

In addition, the Majorana nature of $\nu$ and $N$ imply the existence of novel contributions to the $\mathrm{CP}$ asymmetry in annihilations as well as decays, with a $\mathrm{CP}$ odd phase given by $\operatorname{Im}\left[m M \lambda_{a}^{2}\right]$, see figure 1 . Interestingly enough, this type of contributions requires just one species of real scalars, $S$, and therefore this brings a qualitative difference with typical baryogenesis scenarios. In particular, for the case of decays this means that the particle decaying and the one in the loop can be identical. However these contributions are suppressed by $\frac{m}{2 m_{\chi}}$ (for annihilations) or $\frac{m}{m_{S}}$ (for decays), which is a fairly small factor given that $m \ll T_{\text {sfo }}=\mathcal{O}(100) \mathrm{GeV}$ and $m_{\chi}, m_{S} \gtrsim 1 \mathrm{TeV}$. Whether or not it is possible to have successful baryogenesis with this CP asymmetry is an interesting question, but it is beyond the scope of this work and it would probably require handling very large $\lambda_{1}$ couplings.

The second step in the approximation mentioned above is to analyze how the final helicity asymmetry obtained from the BEs (3.1) and (3.2), $Y_{\Delta \nu}^{f}$, is transferred to the SM lepton sector. This occurs through different fast processes, all of them involving the Yukawa interactions among $\nu$ and the SM leptons. As explained in the introduction, when $m=0$ it is possible to define a lepton number $L$ which is conserved by all these reactions, namely $L=L_{\mathrm{SM}}+L_{\nu}$, where $L_{\mathrm{SM}}$ is the usual lepton number for SM fields, while $\nu^{+}\left(\nu^{-}\right)$is assigned $L_{\nu}=1(-1)$. Then the chemical equilibrium condition for the Yukawa interactions yields $\mu_{\nu}-\mu_{\ell}=\mu_{H}$, where $\mu_{X}$ is the chemical potential of the particle $X$. Taking into account also the whole set of relations among chemical potentials due to all the fast SM processes (including the electroweak sphalerons), the conservation laws, and the relation among chemical potentials and density asymmetries [20,21], one gets that $Y_{B}=a Y_{\Delta \nu}^{f}$. 

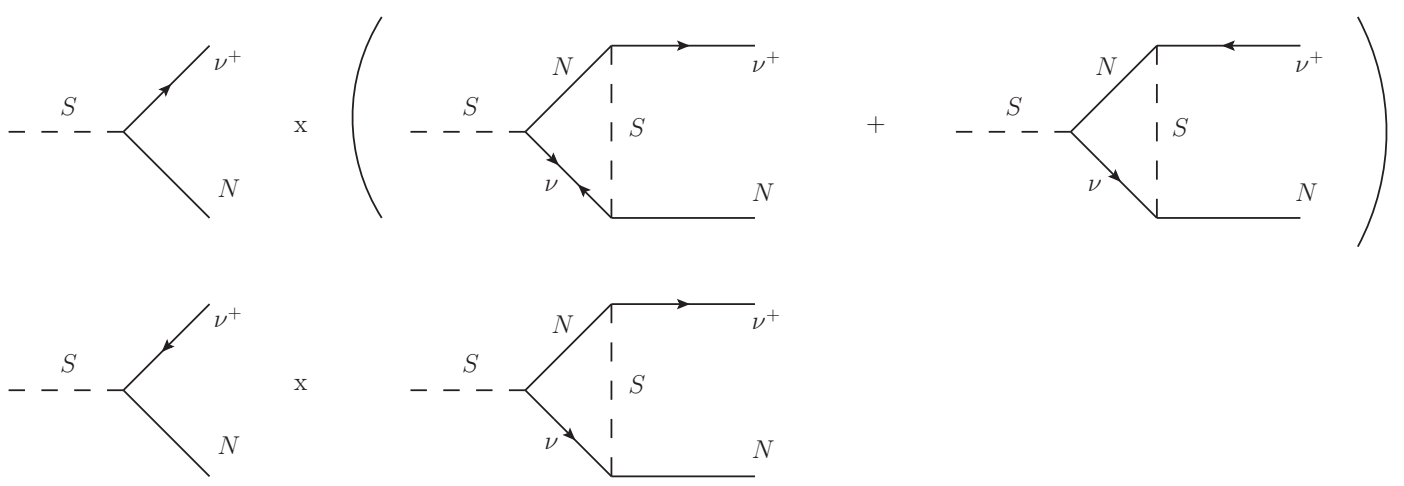

Figure 1. Three of the lowest order contributions to the CP asymmetry in $S$-decays with only one species of scalars. Each one is obtained from the product between a tree level and a one loop diagram. The arrows indicate which of the two operators, $\lambda S \bar{N} P_{R} \nu$ (for ingoing arrows) or $\lambda^{*} S \bar{\nu} P_{L} N$ (for outgoing arrows), is used in each vertex. There are three more contributions obtained from the ones depicted by inverting the arrows in the loops. Each Majorana propagator $(\rightarrow \leftarrow$ or $\longleftrightarrow-)$ as well as a "wrong" helicity emission $\left(\longleftarrow \nu^{+}\right)$brings a suppression $m / m_{S}$ to the $\mathrm{CP}$ asymmetry.

\begin{tabular}{|l|cccc|cccc|}
\hline Parameter & $m_{\chi}$ & $m_{N}$ & $m_{S_{1}}$ & $m_{S_{2}}$ & $\lambda_{\chi 1}$ & $\lambda_{\chi 2}$ & $\lambda_{1}$ & $\lambda_{2}$ \\
\hline Benchmark & 2 & 3 & 6 & 6.6 & 1.5 & 1.65 & 1.7 & 1.8 \\
\hline
\end{tabular}

Table 1. Benchmark point for the WB model. The masses are in $\mathrm{TeV}$ and the phases of the Yukawa couplings have been chosen to maximize the CP asymmetry $\epsilon$. The remaining couplings and masses must be in the ranges specified in section 2 .

Here $a$ is a numerical factor whose value lies between $\sim 1 / 4$ and $\sim 1 / 3$ depending on how many independent fast Yukawa interactions there are among the $\nu_{j}$ and $\ell_{\alpha}$.

Instead, when $m \neq 0, L$ is not conserved. However if $m \ll T$ the rate of processes violating $L$ is suppressed by $(m / T)^{2}$ with respect to the rate of reactions conserving $L$. Hence a net lepton asymmetry can be transferred to the SM sector and baryogenesis can be successful as long as $m \ll T_{\text {sfo }}$.

As an illustration of the allowed parameter space we provide in table 1 a benchmark point that has been obtained solving the BEs (3.1) and (3.2). At the beginning of this section we mentioned that a more accurate calculation of the final baryon asymmetry should take into account that the generation of helicity asymmetry and its partial conversion into net SM lepton and baryon numbers occur simultaneously. Such degree of accuracy is outside the scope of this work, but we note that a convenient way to take into account these spectator effects is to set a $\mathrm{BE}$ for the quantity $B-L_{\mathrm{SM}}-L_{\nu}$.

\section{Other realizations of helicitogenesis}

\subsection{WIMPy leptogenesis with spontaneous $\mathrm{U}(1)_{L}$ symmetry breaking}

A drawback of the minimal model we have described is that there is no justification for the necessary hierarchical spectrum of sterile neutrino masses and couplings. Actually those 
hierarchies would suit very well if the singlet fields were charged under a conserved lepton number. The assignment $L=0,1,1$, and $1 / 2$ to $N_{i}, \nu_{R j}, S_{a}$, and $\chi$, respectively, would imply that $m_{j}=\lambda_{\nu a i j}=\lambda_{N a i j}=h_{N \alpha i}=0$, which perfectly fulfills the requirements of the helicitogenesis mechanism. ${ }^{6}$ The most general $L$-conserving Lagrangian can be written as

$$
\begin{aligned}
-L= & -L_{\mathrm{SM}}-L_{\mathrm{kin}}+V\left(S_{a}, H\right)+m_{\chi} \bar{\chi} \chi+\frac{1}{2} M_{i} \bar{N}_{i} N_{i} \\
& +\frac{1}{2}\left\{\lambda_{\chi a R} S_{a}^{\dagger} \overline{\chi^{c}} P_{R} \chi+\lambda_{\chi a L} S_{a}^{\dagger} \overline{\chi^{c}} P_{L} \chi+\text { h.c. }\right\} \\
& +\left\{\lambda_{a i j} S_{a}^{\dagger} \bar{N}_{i} P_{R} \nu_{j}+h_{\nu \alpha j} \tilde{H} \bar{\ell}_{\alpha} P_{R} \nu_{j}+\text { h.c. }\right\},
\end{aligned}
$$

with

$$
V\left(S_{a}, H\right)=-m_{S a}^{\prime 2} S_{a}^{\dagger} S_{a}+\lambda_{H a b}\left(H^{\dagger} H\right)\left(S_{a}^{\dagger} S_{b}\right)+\lambda_{a b c d}\left(S_{a}^{\dagger} S_{b}\right)\left(S_{c}^{\dagger} S_{d}\right)+\text { h.c. }
$$

The DM field $\chi$ is now a Dirac fermion, and $\chi^{c}=C \bar{\chi}^{T}$. Recall that WB requires at least two scalar fields $S_{a}$. If $m_{S a}^{2}>0$ the complex scalars acquire a non zero vev, lepton number gets spontaneously broken and $\chi$ splits into two Majorana fermions $\chi_{1}, \chi_{2}$ with masses

$$
m_{\chi_{1}, \chi_{2}}=\frac{1}{2}\left\{\mu_{L}+\mu_{R} \pm \sqrt{\left(\mu_{L}-\mu_{R}\right)^{2}+4 m_{\chi}^{2}}\right\}
$$

where $\mu_{L, R} \equiv \sum_{a} \lambda_{\chi a L, \chi a R} u_{a}$ and $u_{a}=\left\langle S_{a}\right\rangle$. Notice that in this model we do not need an additional $Z_{2}$ symmetry, as it is usually the case to avoid the DM decay: the lightest $\chi_{i}$ is stable because of a $Z_{2}$ symmetry which is an unbroken remnant of the global $\mathrm{U}(1)_{L}$, as in $[22]$.

The light neutrino masses would be obtained via a double seesaw [13] mechanism; once the electroweak symmetry is also broken, the SM doublet neutrinos $\nu_{\alpha}$ and the sterile ones, $\nu_{j}, N_{i}$, mix and the mass matrix in the $\left(\nu_{\alpha}, \nu_{j}, N_{i}\right)$ basis becomes:

$$
\mathcal{M}=\left(\begin{array}{ccc}
0 & h_{\nu} v & 0 \\
h_{\nu}^{T} v & 0 & \lambda_{a}^{T} u_{a} \\
0 & \lambda_{a} u_{a} & M
\end{array}\right),
$$

where $v=\langle H\rangle$, the matrix elements of $\lambda_{a}\left(h_{\nu}\right)$ are the Yukawa couplings $\lambda_{a i j}\left(h_{\nu \alpha j}\right)$, and a sum over repeated indices is understood. In the limit $\lambda_{a} u_{a} \ll M$, the singlets $\nu_{j}$ acquire a mass given by

$$
m=\left(\lambda_{a}^{T} u_{a}\right) M^{-1}\left(\lambda_{a} u_{a}\right) \ll M,
$$

while the mass matrix of the three light neutrinos is

$$
m_{L}=h m^{-1} h^{T} v^{2} .
$$

Therefore the smallness of the $\nu_{j}$ masses is due to a seesaw mechanism involving just the SM singlet leptons.

\footnotetext{
${ }^{6}$ In this case the $N_{i}$ can decay before BBN thanks to the large couplings $\lambda_{a i j}$ and the $S_{a}-H$ mixing after electroweak and $\mathrm{U}(1)_{L}$ symmetry breaking.
} 
There are different variants of this scenario, depending on whether lepton number is a global or local symmetry, and the time of spontaneous breaking, denoted by the temperature $T_{L}$. We first consider the case of global $\mathrm{U}(1)_{L}$. If lepton number is broken after the DM freeze out, WB does not work because the $\chi$ field, being charged, can also hold an asymmetry. In turn this asymmetry induces a washout proportional to $\frac{\gamma(\chi \chi \rightarrow N \nu)}{n_{\chi}^{e q} H(z)}$, which freezes out at the same moment as the annihilation of DM, violating one of the basic requirements of $\mathrm{WB}[8] .^{7}$

However, it may be possible that WB occurs via helicitogenesis when $\mathrm{U}(1)_{L}$ is already broken, i.e., $T_{L}>T_{h}>T_{\text {sfo }}$, being $T_{h}$ the temperature at which the $\nu_{j}$ helicity asymmetry is generated. If $T_{L} \sim$ few $\mathrm{TeV}$, it is natural that the physical masses of the singlet scalars $S_{a}$ after $\mathrm{U}(1)_{L}$ breaking satisfy $m_{S a}>m_{\chi_{1}} \gtrsim 1 \mathrm{TeV}$ in a broad region of the parameter space. The sterile neutrinos $\nu_{j}$ acquire a mass given by eq. (4.5), therefore the condition $m_{j} \ll$ $T_{\text {sfo }} \sim 100 \mathrm{GeV}$ needed to generate the helicity asymmetry in $\nu_{j}$, leads to $\left(\lambda_{a} u_{a}\right)^{2} / M \ll$ $100 \mathrm{GeV}$. For instance, assuming $u_{a} \sim M=1 \mathrm{TeV}, \lambda_{a}$ of order 0.2 is required to obtain $m_{j} \sim 40 \mathrm{GeV}$. On the other hand, the Yukawa couplings $\lambda_{a i j}$ should be sizeable, of $\mathcal{O}(1)$, to have enough $\mathrm{CP}$ violation and get the correct DM relic abundance, so there is some tension between these two requirements for WB. Since the neutrino masses depend only on the couplings $\lambda_{a}$ while a combination of $\lambda_{a}$ and $\lambda_{a}^{*}$ appears in the CP-asymmetry, it is conceivable that some cancellations due to phases allow to satisfy all constraints in certain regions of the parameter space with only two singlet scalars. Alternatively, in the presence of three scalars it may happen that $u_{1}, u_{2} \ll u_{3}$ but $\lambda_{1}, \lambda_{2} \gg \lambda_{3 i j}$, achieving a large enough CP asymmetry through the couplings $\lambda_{1,2}$ and getting $m_{j} \ll T_{\text {sfo }}$ without accidental cancellations. Moreover, the vev's at $T_{h}>T_{\text {sfo }}$ may be different from the vev's at $T=0$, as in the singlet Majoron model [23], helping to enlarge the allowed parameter space. We thus conclude that WB seems feasible in the present framework if $T_{L}>T_{h}>T_{\text {sfo. }}$.

The spontaneous breaking of a global symmetry leads to a massless Goldstone boson, the Majoron,

$$
J=\sum_{a} \frac{u_{a}}{u} \operatorname{Im} S_{a}
$$

with $u=\sqrt{\sum_{a} u_{a}^{2}}$. However, non-perturbative gravitational effects are expected to explicitly break global symmetries and provide a mass to the Majoron [24]. If this mass is $m_{J} \lesssim$ few hundred GeV, processes mediated by $N$ such as $\nu_{i}^{+} J \rightarrow \nu_{j}^{-} J$ could lead to a fast washout of the $\nu$ helicity asymmetry and a more detailed analysis is required.

This potential problem can be avoided by promoting lepton number to a gauge symmetry. In this framework, only the case $\mathrm{U}(1)_{B-L}$ is anomaly free without requiring new exotic fermions to cancel anomalies. Then, there are additional constraints due to the searches of the extra $Z^{\prime}$ gauge boson at LEP, Tevatron and LHC. While LHC searches for heavy resonances depend both on the $\mathrm{U}(1)_{B-L}$ coupling strength $g_{B-L}$ and $Z^{\prime}$ mass $[25,26]$, limits

\footnotetext{
${ }^{7}$ The baryon asymmetry is roughly given $Y_{B} \sim \frac{\epsilon}{2}\left[Y_{\chi}\left(z_{w}\right)-Y_{\chi}(\infty)\right]$, where $z_{w}$ is the value of $z$ at which washouts freeze out and $Y_{\chi}(\infty)$ is the relic DM density normalized to the entropy density. Given that $\Omega_{D M} \sim 5 \Omega_{B}$ and $m_{\chi} \gtrsim 1 \mathrm{TeV}$, it is clear that all washout processes must freeze out before DM annihilations, when $Y_{\chi}$ is several orders of magnitude above its final value $Y_{\chi}(\infty)$.
} 
from LEP II imply a model independent bound on the vev $u=M_{Z^{\prime}} /\left(2 g_{B-L}\right) \gtrsim 3 \mathrm{TeV}[27] .{ }^{8}$ Thus in the gauged case $\mathrm{U}(1)_{B-L}$ is necessarily broken before the electroweak phase transition, and the results discussed above for $T_{L}>T_{h}>T_{\text {sfo }}$ apply. Now there is no Majoron, and the only concern would be that the cross sections of the new lepton-number-conserving annihilation channels induced by the $Z^{\prime}$ boson are not too large, so a significant fraction of DM annihilations still proceed through lepton-number-violating processes with $\nu$ in the final state, leading to a sizeable helicity asymmetry in the $\nu$ population. This requirement is easy to satisfy, since $g_{B-L}$ as well as $M_{Z^{\prime}}$ are free parameters.

\subsection{Helicitogenesis in WIMP decay}

It is also possible to realize baryogenesis via helicitogenesis if the matter-antimatter asymmetry does not originate in the DM annihilations, but in the out-of-equilibrium decay of the heavy SM singlets, namely the scalars $S_{a} \rightarrow N_{i} \nu_{j}$, if $m_{S a}>M_{i}$, or the heavy fermions $N_{i} \rightarrow S_{a} \nu_{j}$, if $m_{S a}<M_{i}$. In this case, the conditions $m_{S a}>m_{\chi}$ and $m_{\chi} \lesssim M_{i}<2 m_{\chi}$ are not required. Although the connection between the DM and baryon abundances is in principle lost, it provides a new mechanism to produce the desired helicity asymmetry in the light singlets, $\nu$, so we also discuss this scenario. In the following, we assume that either all the singlet fermions are lighter than the lightest scalar, which we denote by $S_{1}$, or both scalars are lighter than the lightest heavy fermion, denoted by $N_{1}$. These two illustrative scenarios contain all relevant physical features, so more involved mass spectra will not introduce any significant new ingredient in our analysis.

We first consider the possibility that the $\nu_{j}$ helicity asymmetry is generated after $\mathrm{U}(1)_{L}$ breaking, at temperatures $T_{L}>T_{h} \sim M_{d} / 10>T_{\text {sfo }}$, with $M_{d}$ the mass of the decaying particle, $N_{1}$ or $S_{1}$. The following discussion applies to both, global and gauged lepton number, although in the first case if the Majoron is too light it could wash out the helicity asymmetry.

The usual requirements for standard leptogenesis at low temperatures should be satisfied, namely $M_{d} \gtrsim 1 \mathrm{TeV}$ and tiny Yukawa couplings of the decaying particle, typically of order $\lesssim 10^{-7}$. This last requirement has different implications depending on which particle generates the helicity asymmetry, $S_{1}$ or $N_{1}$. In the first case, since the Yukawa couplings $\lambda_{1 i j}$ are tiny, the mass of all the singlets $\nu_{j}$ is mainly generated by the vev and couplings of the scalar $S_{2}$ in eq. (4.5). In the second one, $\lambda_{a 1 j} \lesssim 10^{-7}$ for all $a, j$ leads to a negligible contribution of $N_{1}$ to the $\nu_{j}$ masses, so in order to get at least two massive $\nu$ 's able to generate the observed SM neutrino masses, two more $N$ 's with sizeable Yukawa couplings (of order 0.1) are needed. A big enough CP-asymmetry is obtained for couplings $\gtrsim 10^{-3}$, which is a weaker constrain. If there are only three heavy $N_{i}$, one of the $\nu_{j}$ remains very light, with $m_{j} \lesssim 10^{-11}(u / \mathrm{TeV})^{2} \mathrm{GeV}$, so it should have Yukawa couplings to the SM particles $h_{\nu \alpha j} \ll 10^{-7}$ to avoid too large contributions to the SM neutrino masses. This is not a problem, provided some of the other $\nu$ Yukawa couplings are $\gtrsim 2 \times 10^{-7}$, to efficiently transfer the helicity asymmetry to a lepton number asymmetry in the SM doublets $\ell_{\alpha}$. If there are more than three $N$ 's, all the $\nu$ masses can be $\gtrsim 10 \mathrm{GeV}$.

\footnotetext{
${ }^{8}$ Note that if the $Z^{\prime}$ can decay into sterile neutrinos, the LHC limits may be relaxed [28].
} 
Using the results of [12], we conclude that successful baryogenesis is realized for $m_{S 1} \sim$ few $\mathrm{TeV}, M_{i} \sim(0.5-1) \mathrm{TeV}$ and Yukawa couplings $\lambda_{1 i j} \lesssim 10^{-7}, \lambda_{2 i j} \sim 0.1$. Such values also lead naturally to $m_{j} \sim 10 \mathrm{GeV}$ for $u_{a} \sim M_{i}$. If $m_{S 1}<M_{i}$, leptogenesis could occur in the decay $N_{1} \rightarrow S_{a} \nu_{j}$, for a similar range of masses and couplings of the particles involved, just exchanging the roles of $S_{a}$ and $N_{i}$. This situation seems more contrived, because a largish $M_{i}$ tends to give too small $\nu_{j}$ masses from eq. (4.5), which in turn will produce a light neutrino mass above the cosmological upper limit $\sim 0.3 \mathrm{eV}[1]$. In summary, helicitogenesis via WIMP decay seems likely to occur in a large region of the parameter space if $T_{L}>T_{h}>T_{\text {sfo. }}$. A more exhaustive analysis is beyond the scope of this work.

When $\mathrm{U}(1)_{L}$ breaking takes place at temperatures $T_{L}<T_{h} \sim M_{d} / 10$, baryogenesis occurs in a $B-L$ conserved fashion. Moreover, the $\nu_{j}$ are exactly massless at $T_{h}$, so the requirement $m_{j} \ll T_{\text {sfo }}$ can be relaxed and their helicity asymmetry is in fact a lepton number asymmetry. Generically, we expect $m_{S a} \sim T_{L}$, thus it seems more natural to consider the decay process $N_{1} \rightarrow S_{a} \nu_{j}$. However, since the scalars $S_{a}$ are charged under $\mathrm{U}(1)_{L}$, an equal and opposite lepton number asymmetry is generated in the scalar sector between $S_{a}$ and $S_{a}^{*}$. Therefore this case is not an example of the low scale baryogenesis mechanism proposed here, namely massive decay or annihilation products which do not store asymmetry, such as real scalars or heavy Majorana fermions. In fact, within this model the suppression of washouts due to massive decay products is not effective. There are other possibilities of getting successful leptogenesis, like very heavy neutral leptons, $M_{i} \gtrsim 100 \mathrm{TeV}$, an initial thermal abundance of $N_{1}$ followed by a late decay ${ }^{9}$, or two almost degenerate $N_{i}$ [29]. In general, the neutrino masses $m_{j}$ tend to be too small, unless the scalar vevs are unusually large, $u_{a} \sim M_{i}$, so we do not discuss further this possibility.

\section{Conclusions and outlook}

We have shown how baryogenesis can be achieved from the annihilation or decay of heavy particles - masses $\mathcal{O}(1) \mathrm{TeV}$ - into sterile neutrinos. One of these neutrinos, $N$, must be also "heavy" $(M \gtrsim 0.5 \mathrm{TeV})$, providing a Boltzmann suppression of washouts which can be very fast at these low scales for thermal baryogenesis. The other neutrino, $\nu$, must be relatively "light" ( $m \ll 100 \mathrm{GeV}$ ), so that its mass is negligible during the annihilation or decay epoch, allowing for an helicity asymmetry to be generated. This asymmetry is partially transferred to SM leptons via Yukawa interactions and subsequently reprocessed into a baryon asymmetry via the electroweak sphalerons.

Given that one of the sterile neutrinos must have $m<$ few x $10 \mathrm{GeV}$ and some of the Yukawa couplings must be larger than $h_{\nu} \gtrsim 2 \times 10^{-7}$, the mass of at least one of the light $\mathrm{SM}$ neutrinos must be larger than few $\mathrm{x} 0.01 \mathrm{eV}$-barring phase cancellations in the mass matrix-, which is intriguingly close to the mass scales set by neutrino oscillations.

We have studied a realization of the mechanism in the framework of WB, where there is a relation between the BAU and DM. Since the sterile neutrinos responsible for neutrino

\footnotetext{
${ }^{9}$ An initial thermal abundance of $N_{1}$ can be produced if one of the scalars $S_{a}$ is heavier than $N_{1}$ and has significant Yukawa couplings $\lambda_{a 1 j}$, while the late decay occurs if the Yukawa couplings of $N_{1}$ to the lightest scalar $S_{1}$ are tiny, $\lambda_{11 j} \ll 10^{-7}$.
} 
masses play fundamental roles in the generation of the BAU and the freeze out of DM, helicitogenesis from the annihilation of DM yields a connection between neutrino masses, the BAU and DM.

The required pattern of sterile neutrino masses appears naturally in the so-called double seesaw mechanism, where the smallness of the $\nu$ masses can be due to a $\mathrm{U}(1)_{L}$ symmetry spontaneously broken. Thus, we have constructed an extended $\mathrm{U}(1)_{L}$ symmetric double-seesaw model, including also fermionic DM and two SM singlet scalars, all of them charged under lepton number. We have shown that it is possible to reconcile the helicitogenesis requirements with the measured light neutrino masses provided that $\mathrm{U}(1)_{L}$ breaks spontaneously prior to DM freeze out, or heavy particle decay, and hence before the electroweak phase transition. Within this framework, it seems feasible to have successful WB and explain the observed light neutrino masses with DM and sterile neutrino couplings to the singlet scalars close to $\mathcal{O}(1)$. The suppression of fast washouts is also at work when the helicity asymmetry in the "light" sterile neutrinos $\nu$ is generated during the out-ofequilibrium decay of the heavy states, namely the singlet fermions $N$ or the singlet scalars. In this case, the decaying particle should have tiny Yukawa couplings, to achieve the outof-equilibrium condition at low temperatures, $T \sim \mathcal{O}(1) \mathrm{TeV}$, and the direct connection between the baryon asymmetry and the DM relic abundance is lost. The presence of a light Majoron associated to the breaking of the global $\mathrm{U}(1)_{L}$ symmetry is a potential problem, which can be solved by gauging $\mathrm{U}(1)_{B-L}$.

The mechanism that we propose has certain similarity with baryogenesis via neutrino oscillations, in that the source of the baryonic asymmetry is an helicity asymmetry in the sterile neutrino sector. As a consequence, the three requirements $m \ll 100 \mathrm{GeV}$, fast Yukawa interactions and generation of the helicity asymmetry before sphaleron freeze out apply in all cases, because the first one allows for the existence of the helicity asymmetry itself and the last ones take care of the efficient transfer of the asymmetry to the baryonic sector. However the generation of the $\nu$ helicity asymmetry described here is a completely different process, which involves the CP-violating annihilation or decay of new WIMPs to the sterile neutrinos $\nu$.

Notice that the suppression of the washout processes due to a heavy decay or annihilation product which does not store asymmetry is very general, and not only applies to the scenarios we have described here. E.g., it will also occur if the other decay product is a SM lepton, in which case the lepton asymmetry is generated directly in the out-of-equilibrium decay. This possibility can be realized generalizing the inert doublet model [30] with two inert scalars coupled to SM leptons and sterile neutrinos. Leptogenesis would occur in the decay of the lightest scalar at the $\mathrm{TeV}$ scale, while the second inert scalar is necessary to have $\mathrm{CP}$ violation. Alternatively, the role of the massive decay or annihilation product could also be played by a real scalar.

From the phenomenological point of view, the models that we have discussed involve new particles at the $\mathrm{TeV}$ scale, so in principle they can be tested in current or nearfuture experiments. It would be worth to analyze whether the prospects for detecting the sterile neutrinos, generically very difficult in Type I seesaw models, are improved by their additional interactions with the singlet scalars, which in general mix with the SM Higgs 
field, or with the $Z^{\prime}$ boson in the $\mathrm{U}(1)_{B-L}$ gauged case. With respect to DM detection, the phenomenology of WB models has been extensively analyzed neglecting the mixing among the SM Higgs and the extra scalar singlets $[8,9]$. The observable signatures are not very promising when DM annihilates to leptons, however the impact of the mixing within the scalar sector and of the $Z^{\prime}$ interaction deserves further investigation.

\section{Acknowledgments}

We thank Juan Herrero García, Arcadi Santamaría and Aaron Vincent for illuminating discussions.

This work has been supported by the Spanish MINECO Subprogramme Juan de la Cierva and it has also been partially supported by the Spanish MINECO grants FPA201129678-C02-01 and Consolider-Ingenio CUP (CSD2008-00037), and by Generalitat Valenciana grant PROMETEO/2009/116. In addition we acknowledge partial support from the European Union FP7 ITN INVISIBLES (Marie Curie Actions, PITN-GA-2011-289442).

Open Access. This article is distributed under the terms of the Creative Commons Attribution License (CC-BY 4.0), which permits any use, distribution and reproduction in any medium, provided the original author(s) and source are credited.

\section{References}

[1] Planck collaboration, P.A.R. Ade et al., Planck 2013 results. XVI. Cosmological parameters, Astron. Astrophys. 571 (2014) A16 [arXiv:1303.5076] [INSPIRE].

[2] K. Petraki and R.R. Volkas, Review of asymmetric dark matter, Int. J. Mod. Phys. A 28 (2013) 1330028 [arXiv: 1305.4939] [INSPIRE].

[3] K.M. Zurek, Asymmetric dark matter: theories, signatures and constraints, Phys. Rept. 537 (2014) 91 [arXiv: 1308.0338] [INSPIRE].

[4] J. McDonald, Baryomorphosis: relating the baryon asymmetry to the 'WIMP miracle', Phys. Rev. D 83 (2011) 083509 [arXiv: 1009.3227] [INSPIRE].

[5] J. McDonald, Simultaneous generation of WIMP miracle-like densities of baryons and dark matter, Phys. Rev. D 84 (2011) 103514 [arXiv:1108.4653] [INSPIRE].

[6] S. Davidson and M. Elmer, Similar dark matter and baryon abundances with TeV-scale leptogenesis, JHEP 10 (2012) 148 [arXiv:1208.0551] [INSPIRE].

[7] Y. Cui and R. Sundrum, Baryogenesis for weakly interacting massive particles, Phys. Rev. D 87 (2013) 116013 [arXiv:1212.2973] [INSPIRE].

[8] Y. Cui, L. Randall and B. Shuve, A WIMPy baryogenesis miracle, JHEP 04 (2012) 075 [arXiv: 1112.2704] [INSPIRE].

[9] N. Bernal, F.-X. Josse-Michaux and L. Ubaldi, Phenomenology of WIMPy baryogenesis models, JCAP 01 (2013) 034 [arXiv: 1210.0094] [INSPIRE].

[10] N. Bernal, S. Colucci, F.-X. Josse-Michaux, J. Racker and L. Ubaldi, On baryogenesis from dark matter annihilation, JCAP 10 (2013) 035 [arXiv:1307.6878] [INSPIRE]. 
[11] K. Griest and M. Kamionkowski, Unitarity limits on the mass and radius of dark matter particles, Phys. Rev. Lett. 64 (1990) 615 [InSPIRE].

[12] J. Racker, Mass bounds for baryogenesis from particle decays and the inert doublet model, JCAP 03 (2014) 025 [arXiv: 1308.1840] [INSPIRE].

[13] R.N. Mohapatra and J.W.F. Valle, Neutrino mass and baryon number nonconservation in superstring models, Phys. Rev. D 34 (1986) 1642 [INSPIRE].

[14] E.K. Akhmedov, V.A. Rubakov and A.Y. Smirnov, Baryogenesis via neutrino oscillations, Phys. Rev. Lett. 81 (1998) 1359 [hep-ph/9803255] [INSPIRE].

[15] T. Asaka and M. Shaposhnikov, The $\nu M S M$, dark matter and baryon asymmetry of the universe, Phys. Lett. B 620 (2005) 17 [hep-ph/0505013] [INSPIRE].

[16] B.A. Campbell, S. Davidson, J.R. Ellis and K.A. Olive, On the baryon, lepton flavor and right-handed electron asymmetries of the universe, Phys. Lett. B 297 (1992) 118 [hep-ph/9302221] [INSPIRE].

[17] J.M. Cline, K. Kainulainen and K.A. Olive, Protecting the primordial baryon asymmetry from erasure by sphalerons, Phys. Rev. D 49 (1994) 6394 [hep-ph/9401208] [InSPIRE].

[18] M.C. Gonzalez-Garcia, M. Maltoni and J. Salvado, Robust cosmological bounds on neutrinos and their combination with oscillation results, JHEP 08 (2010) 117 [arXiv:1006.3795] [INSPIRE].

[19] L. Bento and F.C. Santos, Neutrino helicity asymmetries in leptogenesis, Phys. Rev. D 71 (2005) 096001 [hep-ph/0411023] [INSPIRE].

[20] E. Nardi, Y. Nir, J. Racker and E. Roulet, On Higgs and sphaleron effects during the leptogenesis era, JHEP 01 (2006) 068 [hep-ph/0512052] [INSPIRE].

[21] J.A. Harvey and M.S. Turner, Cosmological baryon and lepton number in the presence of electroweak fermion number violation, Phys. Rev. D 42 (1990) 3344 [INSPIRE].

[22] M. Lindner, D. Schmidt and T. Schwetz, Dark matter and neutrino masses from global $\mathrm{U}(1)_{B-L}$ symmetry breaking, Phys. Lett. B 705 (2011) 324 [arXiv:1105.4626] [INSPIRE].

[23] J.M. Cline, G. Laporte, H. Yamashita and S. Kraml, Electroweak phase transition and LHC signatures in the singlet Majoron model, JHEP 07 (2009) 040 [arXiv: 0905. 2559] [INSPIRE].

[24] E.K. Akhmedov, Z.G. Berezhiani, R.N. Mohapatra and G. Senjanović, Planck scale effects on the majoron, Phys. Lett. B 299 (1993) 90 [hep-ph/9209285] [INSPIRE].

[25] CMS collaboration, Search for heavy narrow dilepton resonances in pp collisions at $\sqrt{s}=7 \mathrm{TeV}$ and $\sqrt{s}=8 \mathrm{TeV}$, Phys. Lett. B 720 (2013) 63 [arXiv:1212.6175] [inSPIRE].

[26] ATLAS collaboration, Search for high-mass dilepton resonances in $20 \mathrm{fb}^{-1}$ of pp collisions at $\sqrt{s}=8 \mathrm{TeV}$ with the ATLAS experiment, ATLAS-CONF-2013-017 (2013).

[27] M.S. Carena, A. Daleo, B.A. Dobrescu and T.M.P. Tait, $Z^{\prime}$ gauge bosons at the Tevatron, Phys. Rev. D 70 (2004) 093009 [hep-ph/0408098] [INSPIRE].

[28] A.A. Abdelalim, A. Hammad and S. Khalil, Heavy neutrinos and neutral gauge boson $Z^{\prime}$ at the $L H C$, arXiv: 1405.7550 [INSPIRE].

[29] A. Pilaftsis and T.E.J. Underwood, Electroweak-scale resonant leptogenesis, Phys. Rev. D 72 (2005) 113001 [hep-ph/0506107] [INSPIRE]. 
[30] E. Ma, Verifiable radiative seesaw mechanism of neutrino mass and dark matter, Phys. Rev. D 73 (2006) 077301 [hep-ph/0601225] [INSPIRE]. 